\title{
Gravens by Design: Should Nurturing Stimuli be Limited to Times When a Preterm Baby is Awake?
}

Robert D. White, MD

\section{"For many years, the NICU was an undesirable place for a baby's brain to develop. Lights were bright, the noise was pervasive, painful procedures plentiful, sleep disturbed frequently, and parental access severely restricted."}

For many years, the NICU was an undesirable place for a baby's brain to develop. Lights were bright, the noise was pervasive, painful procedures plentiful, sleep disturbed frequently, and parental access severely restricted. There has been a gradual awakening to the adverse effects this can have on a premature infant who is in the "synaptic explosion" stage of brain growth and development, which, coupled with technological changes that allow us to monitor babies better and less invasively, has enabled us to minimize these noxious stimuli. The importance of infant sleep to brain development has also gained greater recognition so that timing necessary interventions to protect sleep is happening more often.

Concurrently, there has also been a move to introduce nurturing stimuli into the baby's experience. Skin-to-skin care (1), music $(2)$, reading $(3,4)$, and circadian lighting (5) are examples of such stimuli that are much more commonly offered today than they were in the earlier days of NICU care.

"Unfortunately, in many NICUs, the effort to reduce overstimulation and protect infant sleep has come into conflict with the desire to provide nurturing stimuli."

Unfortunately, in many NICUs, the effort to reduce overstimulation and protect infant sleep has come into conflict with the desire to provide nurturing stimuli. It is hypothesized by some practitioners that there is danger in providing these stimuli when an infant is asleep on the grounds that it may cause overstimulation or interfere with the important sleep cycle itself. Thus, nurses and parents are often admonished to stop reading and providing music once their baby falls asleep and to keep the lights dim. Some also prescribe a maximum amount of time these stimuli should be provided in the course of a day, suggesting again that there is a risk of overstimulation. In fact, there is no data to support these beliefs; it is more likely an overly enthusiastic acceptance of the desire to protect babies that has led to this practice - good intentions can have unintended consequences. William Fifer demonstrated that newborns, unlike any other age group, learn while they are asleep $(6,7)$. In utero, we know that infants learn to recognize their mother's voice (8) and smell; we also know that the fetus sleeps most of the time, so these stimuli are likely presented and learned for many hours every day, much of that time while the fetus is asleep.

Unless new data demonstrate that presenting these nurturing stimuli to babies is harmful or that limits are necessary, then we should no longer proscribe their use once an infant falls asleep. Babies are likely to benefit and, in all likelihood, will not be harmed by continuing to provide them with auditory, vestibular, and circadian stimuli throughout the sleep cycle. Of course, these stimuli should be removed if the baby appears to react adversely, but it is much more common to see that babies continue to sleep peacefully even when the nurturing stimuli are continued. Incubators and private rooms are important tools for protecting babies from overstimulation, but they can also become isolation chambers if parents are absent and we do not enrich their sensory environment or do so only in small doses. The best environment for neurosensory development in the NICU is in the arms of a caregiver or when that is not feasible in the presence of nurturing stimuli. Certainly, we need more data to determine what that should look like exactly, but our default should not limit sensory input altogether in a well-meaning effort to protect infant sleep.

\section{"The best environment for neurosensory development in the NICU is in the arms of a caregiver or when that is not feasible in the presence of nurturing stimuli. Certainly, we need more data to determine what that should look like exactly, but our default should not limit sensory input altogether in a well-meaning effort to protect infant sleep."}

NEONATOLOGY TODAY is interested in publishing manuscripts from Neonatologists, Fellows, NNPs and those involved in caring for neonates on case studies, research results, hospital news, meeting announcements, and other pertinent topics.

Please submit your manuscript to: LomaLindaPublishingCompany@gmail.com 


\section{References:}

1. Boundy EO, Dastjerdi R, Spiegelman D, et al. Kangaroo mother care and neonatal outcomes: A metaanalysis. Pediatrics 2016; 137:e20152238.

2. Bieleninik L, Ghetti C, Gold C. Music therapy for preterm infants and their parents: a meta-analysis. Pediatrics 2016; 138:e20160971.

3. Caskey M, Stephens B, Tucker R, Vohr B. Adult talk in the NICU with preterm infants and developmental outcomes. Pediatrics 2014; 133:e578-84.

4. Rubinos $L H$, Brown M, Bahrami, et al. The story behind NICU reading programs. J Perinatol 2016; 36:930-1.

5. Morag I, Ohlsson A. Cycled light in the intensive care unit for preterm and low birth weight infants. Cochrane Database Syst Rev 2016; 2016(8):CD006982.

6. Fifer WP, Byrd DL, Kaku M, et al. Newborn infants learn during sleep. Proc Natl Acad Sci USA 2010; 107:10320-3.

7. Fifer WP, Moon CM. The role of mother's voice in the organization of brain function in the newborn. Acta Paediatr Suppl 1994; 397:86-93.

8. Lee GY, Kisilevsky BS. Fetuses respond to father's voice but prefer mother's voice after birth. Dev Psychobiol 2014; 56:1-11.

Dr. White is a Fellow of the American Academy of Pediatrics, co-chair of the Gravens Conference, chair of the Committee to Establish Recommended Standards for Newborn ICU Design, co-chair of the Newborn Brain Conference, and an adjunct professor at the University of Notre Dame and the Indiana University School of Medicine.

Disclosures: The author has no relevant disclosures

NT

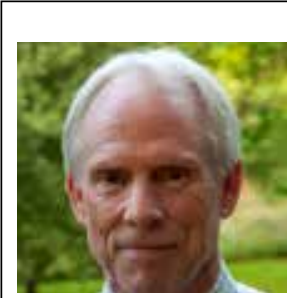

Corresponding Author

Robert D. White, MD

Director, Regional Newborn Program

Beacon Children's Hospital

615 N. Michigan St.

South Bend, IN 46601

Phone: 574-647-7141

Fax: 574-647-3672

Email: Robert White@pediatrix.com

\section{NATIONAL PERINATAL ASSOCIATION}

\section{CORONAVIRUS}

COVID-19

\section{RELIABLE}

RESOURCES:

- CDC: 2019 Novel Coronavirus

- The Lancet: covID-19 and pregnancy

- MotherToBaby: Coronaviruses

- WHO: Emerging respiratory viruses

STAY INFORMED.

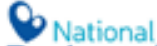
Perinatal

www.nationalperinatal.org

\section{SHARED DECISION-MAKING}

\section{PROTECTS PARENTS + BABIES}

\section{COVID-19}

\section{INFORMED PROVIDERS}

S eek participation

Help explore options

A ssess preferences

$\mathrm{R}$ each a decision

E valuate the decision

CARE DELIVERY REQUIRES

PARTNERSHIP

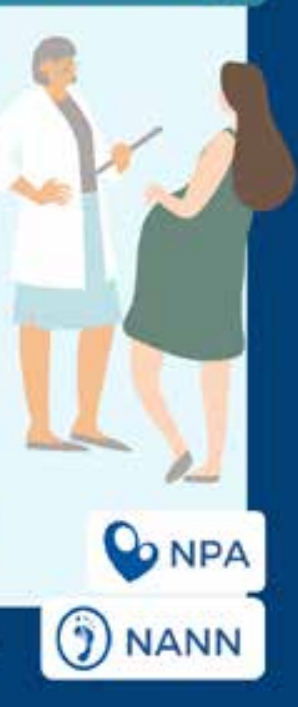

nationalperinatal.org/NPAandNANN

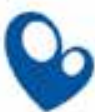

and

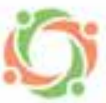

National Perinatal Association and NICU Parent Netwark

mynicunetwork.org 